\title{
Complementation and anti-complementation in intuitionistic anti-fuzzy graphs
}

\author{
R. Muthuraj ${ }^{1}$, V. V. Vijesh ${ }^{2}$ and S. Sujith ${ }^{3}$
}

\begin{abstract}
In this paper, we define the notion of intuitionistic anti-fuzzy graphs and their special cases. We illustrate the concepts regarding properties of intuitionistic anti-fuzzy graphs with some examples. Also, we introduce the concept of complement and anti-complement of intuitionistic anti-fuzzy graphs and prove some of their properties.

\section{Keywords}

Intuitionistic anti-fuzzy graphs, intuitionistic anti-fuzzy subgraphs, $(\mu, \gamma)$-degrees of vertices, strong intuitionistic anti-fuzzy graphs, regular intuitionistic anti-fuzzy graph, complement of intuitionistic anti-fuzzy graphs, anticomplement of intuitionistic anti-fuzzy graphs.

${ }^{1}$ PG \& Research Department of Mathematics, H. H. The Rajah's College, Pudukkottai-622 001, Tamil Nadu, Affiliated to Bharathidasan University, Tiruchirappalli, India.

${ }^{2}$ Department of Mathematics, Sree Narayana Gurukulam College of Engineering, Kolenchery, Ernakulam - 682 311, Kerala, Research Scholar, PG \& Research Department of Mathematics, H. H. The Rajah's College, Pudukkottai, Affiliated to Bharathidasan University,

Tiruchirappalli, Tamil Nadu, India.

${ }^{3}$ Department of Applied Science, College of Engineering, Vadakara, Calicut -673105, Kerala; Co-Guide, PG \& Research Department of Mathematics, H. H. The Rajah's College, Pudukkottai, Affiliated to Bharathidasan University, Tiruchirappalli, Tamil Nadu, India.

Article History: Received 01 October 2019; Accepted 28 December 2019

\section{Contents}

Introduction 883

2

3 885

4 Anti-complement of intuitionistic anti-fuzzy graphs 887

5 Application of intuitionistic anti-fuzzy graph .... 889

6

Conclusion ............................... 890

References .............................. 890

\section{Introduction}

The concept of fuzzy graph was introduced by Kaufmann [3] from the fuzzy relation introduced by Zedah [21]. The study of fuzzy graph theory started in the year 1975 after the phenomenal work published by Rosenfeld [16]. He has introduced another elaborated definition of fuzzy graphs and also proved many results on the fuzzy graph as an analog of graph theory. J. N. Mordeson and Premchand S. Nair [5] introduced the concept of operations on fuzzy graphs, but this concept was extended by M. S. Sunitha and A. Vijayakumar [19]. Muhammad Akram [6] introduced the concept of connected anti fuzzy graphs, self-centroid anti fuzzy graphs constant and totally constant anti fuzzy graphs with some of their properties together with regularity and irregularity. R. Seethalakshmi and R. B. Gnanajothi [17] introduced the concept of anti-fuzzy graph and discussed the concept of some operations such as union and join on anti-fuzzy graphs.

Intuitionistic fuzzy sets are generalization of fuzzy sets [21]. Atanassov [1] introduced the concept of intuitionistic fuzzy relation, which has both membership grades and nonmembership grades. He applied his ideas into expert systems, pattern recognition and mainly in decision making. A new emerging study of an intuitionistic fuzzy graph (IFG) has been addressed in [18]. The operations [15] and particular cases of intuitionistic fuzzy graphs [14] were done by Parvathy and Karunabigai. Whenever we discuss the intuitionistic fuzzy structures in any algebraic theory, analogously the notion of intuitionistic anti-fuzzy structures has been studied. However, in the theory of intuitionistic fuzzy graphs, no theory on intuitionistic anti-fuzzy structures has been introduced. This motivated us to introduce the theory of intuitionistic anti-fuzzy graphs [10]. R. Muthuraj, Vijesh V. V. and Sujith S. [12] explained the concept of split and strong split dominations in intuitionistic anti-fuzzy graphs.

In this paper, we introduce the concept of complement of intuitionistic anti-fuzzy graphs and anti-complement on 
intuitionistic anti-fuzzy graphs. We derive some theorems and results on these two idea together with an application of intuitionistic anti-fuzzy graph.

\section{Preliminaries}

Definition 2.1. An intuitionistic anti-fuzzy graph is of the form $G=\langle V, E\rangle$ where

(i) $V=\left\{v_{1}, v_{2}, \ldots v_{n}\right\}$ such that $\mu_{1}: V \rightarrow[0,1]$ and $\gamma_{1}$ : $V \rightarrow[0,1]$ denote the degree of membership and nonmembership of the element $v_{i} \in V$ respectively and

$$
0 \leq \mu_{1}\left(v_{i}\right)+\gamma_{1}\left(v_{i}\right) \leq 1
$$

for every $v_{i} \in V,(i=1,2, \ldots n)$,

(ii) $E \subseteq V \times V$ where $\mu_{2}: V \times V \rightarrow[0,1]$ and $\gamma_{2}: V \times V \rightarrow$ $[0,1]$ are such that

$$
\begin{aligned}
\mu_{2}\left(v_{i}, v_{j}\right) & \geq \max \left\{\mu_{1}\left(v_{i}\right), \mu_{1}\left(v_{j}\right)\right\}, \\
\gamma_{2}\left(v_{i}, v_{j}\right) & \geq \min \left\{\gamma_{1}\left(v_{i}\right), \gamma_{1}\left(v_{j}\right)\right\} \\
0 & \leq \mu_{2}\left(v_{i}, v_{j}\right)+\gamma_{2}\left(v_{i}, v_{j}\right) \leq 1 \ldots
\end{aligned}
$$

for every $\left(v_{i}, v_{j}\right) \in E,(i, j=1,2, \ldots n)$.

Note 2.2. If one of the inequalities (2.1) or (2.2) or (2.3) or (2.4) is not satisfied, then the graph $G$ is not an intuitionistic anti-fuzzy graph.

Note 2.3. An intuitionistic anti-fuzzy graph $\langle V, E\rangle$ is denoted by $G_{A}\langle V, E\rangle$.

Example 2.4. See Fig. 1.

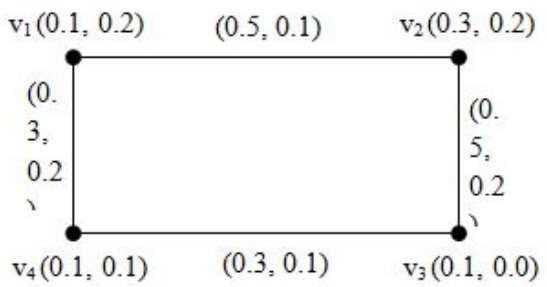

Figure 1. Intuitionistic anti-fuzzy graph $G_{A}\langle V, E\rangle$

Definition 2.5. An intuitionistic anti-fuzzy $\operatorname{graph}_{A}\left(V^{\prime}, E^{\prime}\right)$ is an intuitionistic anti-fuzzy sub graph of $G_{A}\langle V, E\rangle$ if $V^{\prime} \subseteq$ $V, E^{\prime} \subseteq$ Esuch that $\mu_{1 i}^{\prime} \leq \mu_{1 i}, \quad \gamma_{1 i}^{\prime} \geq \gamma_{1 i}$ and $\mu_{2 i j}^{\prime} \leq \mu_{2 i j}, \gamma_{2 i j}^{\prime} \geq$ $\gamma_{2 i j}$.

Definition 2.6. An intuitionistic anti-fuzzy sub graph $H_{A}\left(V^{\prime}, E^{\prime}\right)$ is called a spanning intuitionistic anti-fuzzy sub graph of $G_{A}\langle V, E\rangle$ if

(i) $V^{\prime}=V, E^{\prime}=E$

(ii) $\mu_{1 i}^{\prime}=\mu_{1 i}, \quad \gamma_{1 i}^{\prime}=\gamma_{1 i}, \forall i, j$

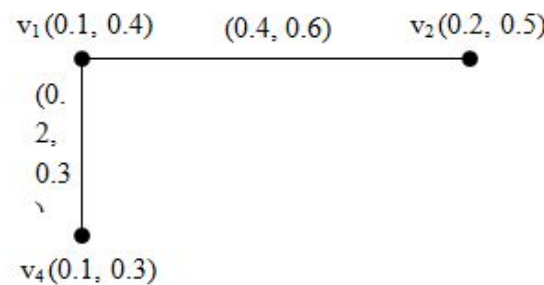

Figure 2. Intuitionistic anti-fuzzy sub graph of $G_{A}\langle V, E\rangle$

Example 2.7. Consider the intuitionistic anti-fuzzy graph $G$ ${ }_{A}\langle V, E\rangle$ given in example 2.1. The intuitionistic anti-fuzzy sub graph $H_{A}\left(V^{\prime}, E^{\prime}\right)$ is obtained in Fig. 2.

Definition 2.8. Let $G_{A}=\langle V, E\rangle$ be an intuitionistic anti-fuzzy graph. Then the vertex cardinality of $V$ is defined by

$$
|V|=\sum_{v_{i} \in V}\left(\frac{1+\mu_{1}\left(v_{i}\right)-\gamma_{1}\left(v_{i}\right)}{2}\right)
$$

Definition 2.9. Let $G_{A}=\langle V, E\rangle$ be an intuitionistic anti-fuzzy graph. Then the edge cardinality of Eis defined by

$$
\begin{aligned}
|E| & =\sum_{\left(v_{i}, y_{j}\right) \in E}\left(\frac{1+\mu_{2}\left(v_{i}, v_{j}\right)-\gamma_{2}\left(v_{i}, v_{j}\right)}{2}\right) \\
& =\sum_{e_{i} \in E}\left(\frac{1+\mu_{2}\left(e_{i}\right)-\gamma_{2}\left(e_{i}\right)}{2}\right)
\end{aligned}
$$

Definition 2.10. Let $G_{A}=\langle V, E\rangle$ be an intuitionistic antifuzzy graph. Then the cardinality of $G_{A}$ is defined by

$$
\begin{aligned}
\left|G_{A}\right|=|| V|+| E||=\mid \sum_{v_{i} \in V}\left(\frac{1+\mu_{1}\left(v_{i}\right)-\gamma_{1}\left(v_{i}\right)}{2}\right) \\
+\sum_{\left(v_{i}, v_{j}\right) \in E}\left(\frac{1+\mu_{2}\left(v_{i}, v_{j}\right)-\gamma_{2}\left(v_{i}, v_{j}\right)}{2}\right) \mid
\end{aligned}
$$

Definition 2.11. Let $G_{A}=\langle V, E\rangle$ be an intuitionistic antifuzzy graph. The $(\mu, \gamma)$-degree of a vertex $u$ is defined by a sum of weights of the edges that are incident at $u$ and it is denoted by $d_{G_{A}}(u)$ ie, $d_{G_{A}}(u)=\left(d_{\mu}(u), d_{\gamma}(u)\right)$, where $d_{\mu}(u)=\sum_{v \neq u} \mu_{2}(u, v)$ and $d_{\gamma}(u)=\sum_{v \neq u} \gamma_{2}(u, v)$.

Definition 2.12. The minimum $(\mu, \gamma)$-degree of an intuitionistic anti-fuzzy graph $G_{A}=\langle V, E\rangle$ is $\delta\left(G_{A}\right)=\left(\delta_{\mu}\left(G_{A}\right), \delta_{\gamma}\left(G_{A}\right)\right)$, where $\delta_{\mu}\left(G_{A}\right)=\min \left\{d_{\mu}(v) / v \in V\right\}$ and

$$
\delta_{\gamma}\left(G_{A}\right)=\min \left\{d_{\gamma}(v) / v \in V\right\} .
$$

Definition 2.13. The maximum $(\mu, \gamma)$-degree of an intuitionistic anti-fuzzy graph $G_{A}=\langle V, E\rangle$ is

$$
\Delta\left(G_{A}\right)=\left(\Delta_{\mu}\left(G_{A}\right), \Delta_{\gamma}\left(G_{A}\right)\right),
$$

where $\Delta_{\mu}\left(G_{A}\right)=\max \left\{d_{\mu}(v) / v \in V\right\}$ and

$$
\Delta_{\gamma}\left(G_{A}\right)=\max \left\{d_{\gamma}(v) / V \in V\right\}
$$




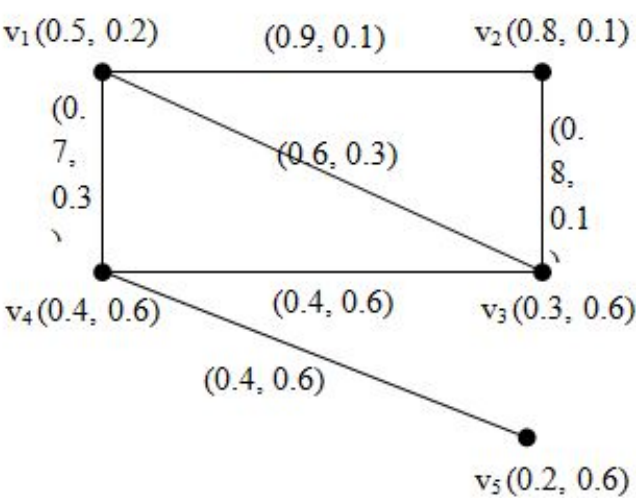

Figure 3. Intuitionistic anti-fuzzy graph $G_{A}\langle V, E\rangle$

Example 2.14. Consider the following intuitionistic antifuzzy graph. See Fig. 3.

Vertex cardinality of $V$ is

$$
|V|=\sum_{v_{i} \in V}\left(\frac{1+\mu_{1}\left(v_{i}\right)-\gamma_{1}\left(v_{i}\right)}{2}\right)=2.55
$$

Edge cardinality of $E$ is

$$
|E|=\sum_{\left(v_{1}, v_{j}\right) \in E}\left(\frac{1+\mu_{2}\left(v_{i}, y_{j}\right)-\gamma_{2}\left(v_{i}, y_{j}\right)}{2}\right)=3.9
$$

Cardinality of $G_{A}$ is $\left|G_{A}\right|=|| V|+| E||=6.45$. Now, $(\mu, \gamma)$ degrees of vertices $v_{i}$ are $d_{G_{a}}\left(v_{1}\right)=(2.2,0.7), d_{G_{A}}\left(v_{2}\right)=$ $(1.7,0.2), d_{G_{A}}\left(v_{3}\right)=(1.8,1.0), d_{G_{A}}\left(v_{4}\right)=(1.5,1.5), d_{G_{A}}\left(v_{5}\right)$ $=(0.4,0.6)$.

Thus, minimum $(\mu, \gamma)$-degree of $G_{A}$ is

$$
\delta\left(G_{A}\right)=\left(\delta_{\mu}\left(G_{A}\right), \delta_{\gamma}\left(G_{A}\right)\right)=(0.4,0.2) .
$$

Maximum $(\mu, \gamma)$-degree of $G_{A}$ is

$$
\Delta\left(G_{A}\right)=\left(\Delta_{\mu}\left(G_{A}\right), \Delta_{\gamma}\left(G_{A}\right)\right)=(2.2,1.5)
$$

Definition 2.15. An edge $e=(u, v)$ of intuitionistic anti-fuzzy graph $G_{A}=\langle V, E\rangle$ is said to be an effective edge if $\mu_{2}(u, v)=$ $\max \left\{\mu_{1}(u), \mu_{1}(v)\right\}$ and $\gamma_{2}(u, v)=\min \left\{\gamma_{1}(u), \gamma_{1}(v)\right\}$.

Definition 2.16. An intuitionistic anti-fuzzy graph $G_{A}=\langle V, E\rangle$ is said to be complete if $\mu_{2 i j}=\max \left\{\mu_{1 i}, \mu_{1 j}\right\}$ and $\gamma_{2 i j}=$ $\min \left\{\gamma_{1 i}, \gamma_{1 j}\right\}, \forall v_{i}, v_{j} \in V$.

Note 2.17. The underlying graph of a complete intuitionistic anti-fuzzy graph is complete.

Example 2.18. The graph given below is a complete intuitionistic anti-fuzzy graph. See Fig. 4.

Definition 2.19. An intuitionistic anti-fuzzy $\operatorname{graph} G_{A}\langle V, E\rangle$ is said to be strong if $\mu_{2 i j}=\max \left\{\mu_{1 i}, \mu_{1 j}\right\}$ and

$$
\gamma_{2 i j}=\min \left\{\gamma_{1 i}, \gamma_{1 j}\right\}, \forall\left(v_{i}, v_{j}\right) \in E \text {. }
$$

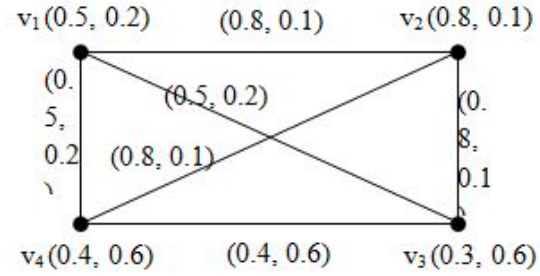

Figure 4. Complete intuitionistic anti-fuzzy graphG ${ }_{A}\langle V, E\rangle$

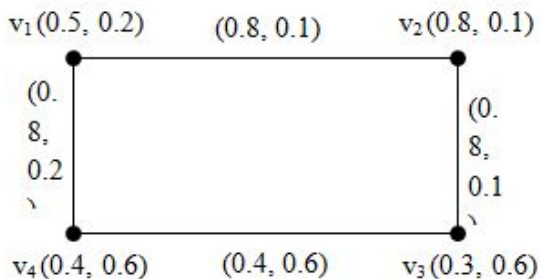

Figure 5. Strong intuitionistic anti-fuzzy graphG ${ }_{A}\langle V, E\rangle$

Example 2.20. The graph given below is a strong intuitionistic anti-fuzzy graph. See Fig. 5.

Definition 2.21. An intuitionistic anti-fuzzy $\operatorname{graph}_{A}\langle V, E\rangle$ is said to be $a\left(K_{1}, K_{2}\right)$-regular if $d_{G_{A}}\left(v_{i}\right)=\left(K_{1}, K_{2}\right), \forall v_{i} \in$ Vand also $G_{A}$ is said to be a regular intuitionistic anti-fuzzy graph of $(\mu, \gamma)$-degree $\left(K_{1}, K_{2}\right)$, where $K_{1}$ and $K_{2}$ are real constants.

Example 2.22. The graph given below is a $(1.3,0.6)$-regular intuitionistic anti-fuzzy graph. See Fig. 6.

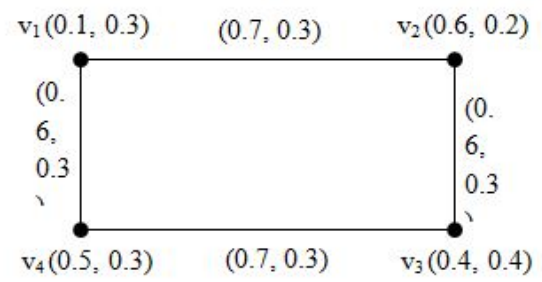

Figure 6. Regular intuitionistic anti-fuzzy graph $G_{A}\langle V, E\rangle$

\section{Complement of strong intuitionistic anti-fuzzy graph}

Definition 3.1. The complement of a strong intuitionistic antifuzzy graph $G_{A}=\langle V, E\rangle$ is a strong intuitionistic anti-fuzzy graph $\overline{G_{A}}=\langle\bar{V}, \bar{E}\rangle$, where

(i) $\bar{V}=V$

(ii) $\overline{\mu_{11}}=\mu_{1 i}$ and $\overline{\gamma_{11}}=\gamma_{1 i}, \forall i=1,2,3, \ldots, n$ 
(iii)

$$
\begin{aligned}
\overline{\mu_{21}} & = \begin{cases}0, & \text { if } \mu_{2 i j}>0 \\
\max \left\{\mu_{1 i}, \mu_{1 j}\right\}, & \text { if } \mu_{2 i j}=0\end{cases} \\
& =\max \left\{\mu_{1 i}, \mu_{1 j}\right\}-\mu_{2 i j}, \text { for all } i, j=1,2, \ldots, n \\
\overline{\gamma_{211}} & = \begin{cases}0, & \text { if } \quad \gamma_{2 i j}>0 \\
\min \left\{\gamma_{1 i}, \gamma_{1 j}\right\}, & \text { if } \gamma_{2 i j}=0\end{cases} \\
& =\min \left\{\gamma_{1 i}, \quad \gamma_{1 j}\right\}-\gamma_{2 i j}, \text { for all } i, j=1,2, \ldots, n
\end{aligned}
$$

Example 3.2. See Fig. 7. and 8 The complement of this

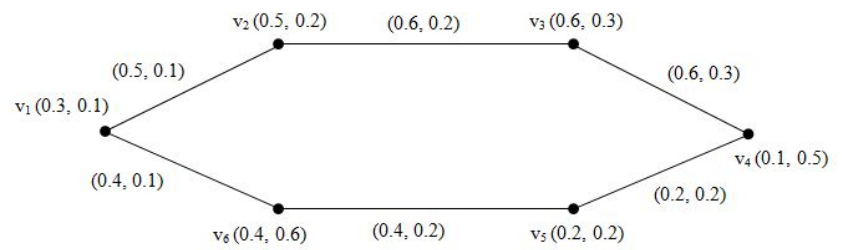

Figure 7. Strong Intuitionistic anti-fuzzy graph $\mathrm{G}_{\mathrm{A}}$

Strong Intuitionistic anti-fuzzy graph $G_{A}$ is given below:

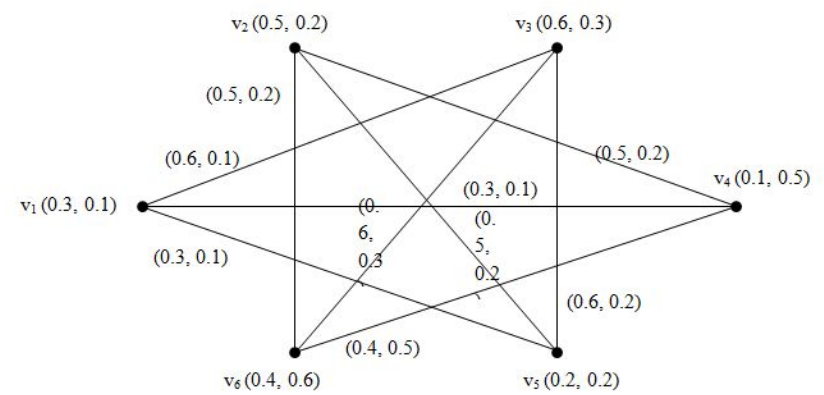

Figure 8. Complement Strong Intuitionistic anti-fuzzy graph $\overline{G_{A}}$

Theorem 3.3. The edge set of the complement of a complete intuitionistic anti-fuzzy graph $G_{A}=\langle V, E\rangle$ is empty. That is, if $G_{A}=\langle V, E\rangle$ is a complete intuitionistic anti-fuzzy graph and $\overline{G_{A}}=\langle\bar{V}, \bar{E}\rangle$ is its complement intuitionistic anti-fuzzy graph then, $\bar{E}=\varphi$.

Proof. Let $G_{A}=\langle V, E\rangle$ be a completeintuitionistic anti-fuzzy graph. Therefore,

$$
\begin{aligned}
\mu_{2}(u, v) & =\max \left\{\mu_{1}(u), \mu_{1}(v)\right\} \\
\gamma_{2}(u, v) & =\min \left\{\gamma_{1}(u), \gamma_{1}(v)\right\}, \text { for all } u, v \in V .
\end{aligned}
$$

Let $\overline{G_{A}}=\langle\bar{V}, \bar{E}\rangle$ be the complement of $G_{A}$. Then, $\overline{\mu_{1}}(u)=$ $\mu_{1}(u) ; \overline{\gamma_{1}}(u)=\gamma_{1}(u), \forall u \in V$. Thus, for all $u, v \in V$,

$$
\begin{aligned}
\overline{\mu_{2}}(u, v) & =\max \left\{\mu_{1}(u), \mu_{1}(v)\right\}-\mu_{2}(u, v) \\
& =\max \left\{\mu_{1}(u), \mu_{1}(v)\right\}-\max \left\{\mu_{1}(u), \mu_{1}(v)\right\} \\
& =0 \\
\overline{\gamma_{2}}(u, v) & =\min \left\{\gamma_{1}(u), \gamma_{1}(v)\right\}-\gamma_{2}(u, v) \\
& =\min \left\{\gamma_{1}(u), \gamma_{1}(v)\right\}-\min \left\{\gamma_{1}(u), \gamma_{1}(v)\right\} \\
& =0
\end{aligned}
$$

So, $\left(\overline{\mu_{2}}(u, v), \bar{\gamma}_{2}(u, v)\right)=(0,0), \quad \forall u, v \in V$.

This means, there does not exists arcs between any two distinct vertices $u$ and $v$ of $\overline{G_{A}}$. Thus, $\bar{E}=\varphi$.

Remark 3.4. The complement of a strong intuitionistic antifuzzy graph is again a strong intuitionistic anti-fuzzy graph.

Example 3.5. See Fig. 9. and 10.

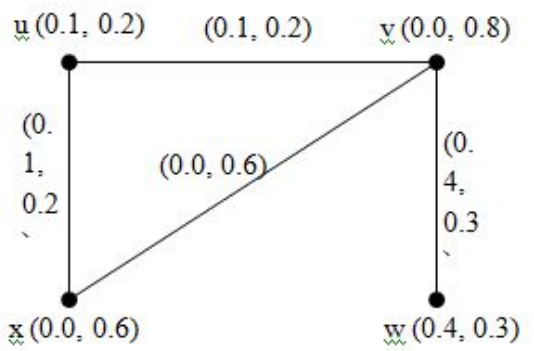

Figure 9. Strong Intuitionistic anti-fuzzy graph $G_{A}$

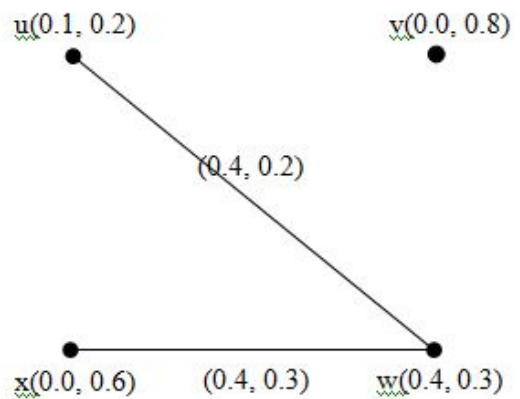

Figure 10. Complement Strong Intuitionistic anti-fuzzy graph $\bar{G}_{A}$.

Here, figure 9 is a Strong Intuitionistic anti-fuzzy graph $G_{A}$ and figure 10 is its complement, which is also a strong graph as well an intuitionistic anti-fuzzy graph.

Theorem 3.6. The complement of complement of a strong intuitionistic anti-fuzzy graph $G_{A}=\langle V, E\rangle$ is the same IAFG $G_{A}$ itself. $\left(i e^{\overline{G_{A}}}=G_{A}\right)$

Proof. Let $G_{A}=\langle V, E\rangle$ be a strong intuitionistic anti-fuzzy graph. Then,

$$
\begin{aligned}
\mu_{2}(u, v) & =\max \left\{\mu_{1}(u), \mu_{1}(v)\right\} \\
\gamma_{2}(u, v) & =\min \left\{\gamma_{1}(u), \gamma_{1}(v)\right\}, \text { for all }(u, v) \in E .
\end{aligned}
$$

Let $\overline{G_{A}}=\langle\bar{V}, \bar{E}\rangle$ be the complement of $G_{A}$. Then,

$$
\begin{aligned}
\overline{\mu_{1}}(u) & =\mu_{1}(u) ; \quad \overline{\gamma_{1}}(u)=\gamma_{1}(u), \quad \forall u \in V \\
\overline{\mu_{2}}(u, v) & =\max \left\{\mu_{1}(u), \mu_{1}(v)\right\}-\mu_{2}(u, v) \\
\overline{\gamma_{2}}(u, v) & =\min \left\{\gamma_{1}(u), \gamma_{1}(v)\right\}-\gamma_{2}(u, v), \text { for all }(u, v) \in E .
\end{aligned}
$$


But in the complement $\overline{\bar{G}} \bar{A}\langle\overline{\bar{V}}, \overline{\bar{E}}\rangle$

$$
\begin{aligned}
\overline{\overline{\mu_{1}}}(u)= & \overline{\mu_{1}}(u)=\mu_{1}(u) ; \quad \overline{\overline{\gamma_{1}}}(u)=\overline{\gamma_{1}}(u)=\gamma_{1}(u), \quad \forall u \in V \\
\overline{\mu_{2}}(u, v) & =\max \left\{\overline{\mu_{1}}(u), \overline{\mu_{1}}(v)\right\}-\overline{\mu_{2}}(u, v) \\
& =\max \left\{\overline{\mu_{1}}(u), \overline{\mu_{1}}(v)\right\}-\left[\max \left\{\mu_{1}(u), \mu_{1}(v)\right\}-\mu_{2}(u, v)\right] \\
& =\max \left\{\mu_{1}(u), \mu_{1}(v)\right\}-\max \left\{\mu_{1}(u), \mu_{1}(v)\right\}+\mu_{2}(u, v) \\
& =\mu_{2}(u, v) \\
\overline{\overline{\gamma_{2}}}(u, v) & =\min \left\{\overline{\gamma_{1}}(u), \overline{\gamma_{1}}(v)\right\}-\overline{\gamma_{2}}(u, v) \\
& =\min \left\{\bar{\gamma}(u), \overline{\gamma_{1}}(v)\right\}-\left[\min \left\{\gamma_{1}(u), \gamma_{1}(v)\right\}-\gamma_{2}(u, v)\right] \\
& =\min \left\{\gamma_{1}(u), \gamma_{1}(v)\right\}-\min \left\{\gamma_{1}(u), \gamma_{1}(v)\right\}+\gamma_{2}(u, v) \\
& =\gamma_{2}(u, v)
\end{aligned}
$$

Hence, $\overline{\overline{G_{A}}}=G_{A}$.

Theorem 3.7. Let $G_{A}=\langle V, E\rangle$ be a strong intuitionistic antifuzzy graph and $\overline{G_{A}}=\langle\bar{V}, \bar{E}\rangle$ be its complement strong intuitionistic anti-fuzzy graph. Then, the anti-union $G_{A} \cup \overline{G_{A}}$ is always a complete intuitionistic anti-fuzzy graph.

Proof. Let $G_{A}=\langle V, E\rangle$ be a strong intuitionistic anti-fuzzy graph. Then.

$$
\begin{aligned}
\mu_{2}(u, v) & =\max \left\{\mu_{1}(u), \mu_{1}(v)\right\} \\
\gamma_{2}(u, v) & =\min \left\{\gamma_{1}(u), \gamma_{1}(v)\right\}, \text { for all }(u, v) \in E
\end{aligned}
$$

Let $\overline{G_{A}}=\langle\bar{V}, \bar{E}\rangle$ be the complement strong intuitionistic antifuzzy graph. Then,

$$
\begin{aligned}
& \overline{\mu_{11}}=\mu_{1 i} ; \quad \overline{\gamma_{11}}=\gamma_{1 i}, \quad \forall i=1,2,3, \ldots, n \\
& \overline{\mu_{21}}=\max \left\{\mu_{1 i}, \quad \mu_{1 j}\right\}-\mu_{2 i j}, \text { for all } i, j=1,2, \ldots, n \\
& \overline{\gamma_{21]}}=\min \left\{\gamma_{1 i}, \gamma_{1 j}\right\}-\gamma_{2 i j}, \text { for all } i, j=1,2, \ldots, n
\end{aligned}
$$

If $(u, v) \in E$, then

$$
\begin{aligned}
& \overline{\mu_{2}}(u, v)= \max \left\{\mu_{1}(u), \mu_{1}(v)\right\}-\mu_{2}(u, v) \\
&= \max \left\{\mu_{1}(u), \mu_{1}(v)\right\}-\max \left\{\mu_{1}(u), \mu_{1}(v)\right\} \\
&= 0 \quad\left(\text { Since, } G_{A}\right. \text { is a strong intuitionistic } \\
&\quad \text { anti-fuzzy graph. }) \\
& \overline{\gamma_{2}}(u, v)=\min \left\{\gamma_{1}(u), \gamma_{1}(v)\right\}-\gamma_{2}(u, v) \min \left\{\gamma_{1}(u), \gamma_{1}(v)\right\}-\min \left\{\gamma_{1}(u), \gamma_{1}(v)\right\} \\
&=0 \quad\left(\text { Since, } G_{A}\right. \text { is a strong intuitionistic } \\
&\quad \text { anti-fuzzy graph. })
\end{aligned}
$$

Thus, for all $(u, v) \in E$ of $G_{A}=\langle V, E\rangle$

$$
\left(\overline{\mu_{2}}(u, v), \bar{\gamma}_{2}(u, v)\right)=(0,0) \text { in } \overline{G_{A}} .
$$

That is, there does not exist an arc between vertices $u$ and $v$ in complement IAFG $\overline{G_{A}}$. If $(u, v) \notin E$, then

$$
\begin{aligned}
\overline{\mu_{2}}(u, v) & =\max \left\{\mu_{1}(u), \mu_{1}(v)\right\}-\mu_{2}(u, v) \\
& =\max \left\{\mu_{1}(u), \mu_{1}(v)\right\} \\
\overline{\gamma_{2}}(u, v) & =\min \left\{\gamma_{1}(u), \gamma_{1}(v)\right\}-\gamma_{2}(u, v) \\
& =\min \left\{\gamma_{1}(u), \gamma_{1}(v)\right\}
\end{aligned}
$$

Thus, for all $(u, v) \notin E$ of $G_{A}=\langle V, E\rangle$,

$$
\begin{aligned}
& \overline{\mu_{2}}(u, v)=\max \left\{\mu_{1}(u), \mu_{1}(v)\right\} \\
& \overline{\gamma_{2}}(u, v)=\min \left\{\gamma_{1}(u), \gamma_{1}(v)\right\}, \text { for all }(u, v) \in \bar{E} \text { in } \overline{G_{A}} .
\end{aligned}
$$

So, the vertices which are not incident in $G_{A}$ are incident vertices in $\overline{G_{A}}$ and the corresponding arcs are effective arcs.

When we find the anti-union of $G_{A}$ and $\overline{G_{A}}$, the vertex set of $G_{A} \cup \overline{G_{A}}$ is same as $V$ itself with the same membership and non-membership values as they are in $G_{A}\left(\right.$ or in $\left.\overline{G_{A}}\right)$. The edge membership and non-membership values of an arbitrary edge $(u, v)$ in anti-union $G_{A} \cup \overline{G_{A}}$ because

$$
\begin{array}{r}
\left(\mu_{2} \cup \overline{\mu_{2}}\right)(u, v)= \begin{cases}\mu_{2}(u, v), & \text { if }(u, v) \in E \backslash \bar{E} \\
\overline{\mu_{2}}(u, v), & \text { if }(u, v) \in \bar{E} \backslash E\end{cases} \\
\left(\gamma_{2} \cup \overline{\gamma_{2}}\right)(u, v)= \begin{cases}\gamma_{2}(u, v), & \text { if }(u, v) \in E \backslash \bar{E} \\
\overline{\gamma_{2}}(u, v), & \text { if }(u, v) \in \bar{E} \backslash E\end{cases}
\end{array}
$$

Thus, $G_{A} \cup \overline{G_{A}}$ gives its underlying graph as a complete graph, where $G_{A}$ and $\overline{G_{A}}$ are strong intuitionistic anti-fuzzy graphs.

Since the anti-union of two strong intuitionistic anti-fuzzy graphs is also a strong intuitionistic antifuzzy graph, $G_{A} \cup \overline{G_{A}}$ becomes a strong intuitionistic anti-fuzzy graph.

Hence, $G_{A} \cup \overline{G_{A}}$ will be a complete intuitionistic antifuzzy graph.

\section{Anti-complement of intuitionistic anti-fuzzy graphs}

Definition 4.1. The anti-complement of an intuitionistic antifuzzy graph $G_{A}=\langle V, E\rangle$ is a graph $\widetilde{G_{A}}=(\tilde{V}, \widetilde{E})$ where

(i) $\widetilde{V}=V$

(ii) $\widetilde{\mu_{11}}=\mu_{1 i}$ and $\widetilde{\gamma_{11}}=\gamma_{1 i}, \forall i=1,2,3, \ldots, n$

(iii) $\widetilde{\mu_{21 j}}=1-\mu_{2 i j}+\max \left\{\mu_{1 i}, \mu_{1 j}\right\}$ and $\widetilde{\gamma_{21 j}}=1-\gamma_{2 i j}+$ $\min \left\{\gamma_{1 i}, \gamma_{1 j}\right\}, \forall\left(v_{i}, v_{j}\right) \in E$.

Example 4.2. See Fig. 11. and 12 .

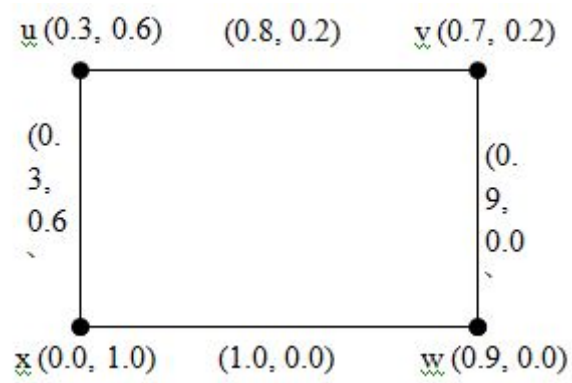

Figure 11. Intuitionistic anti-fuzzy graph $G_{A}$

Proposition 4.3. The anti-complement of an intuitionistic anti-fuzzy graph need not be an intuitionistic anti-fuzzy graph. 


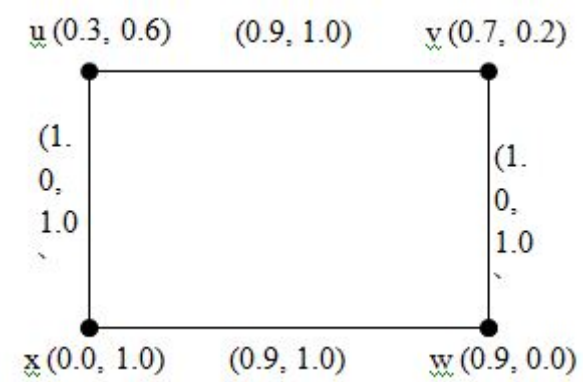

Figure 12. Anti-complement graph $\left(G_{A}\right)$

In the above example $4.2, G_{A}$ in figure 11 is an intuitionistic anti fuzzy graph, but $\widetilde{G_{A}}$ in figure 12 is not an intuitionistic anti-fuzzy graph, since which does not satisfy the condition

$$
0 \leq \mu_{2}\left(v_{i}, v_{j}\right)+\gamma_{2}\left(v_{i}, v_{j}\right) \leq 1
$$

Theorem 4.4. If $G_{A}$ is a complete intuitionistic anti-fuzzy graph, then its anti-complement $\widetilde{G_{A}}$ is $(n-1, n-1)$-regular.

Proof. Let $G_{A}=\langle V, E\rangle$ be a complete intuitionistic anti-fuzzy graph.

$$
\therefore \mu_{2 i j}=\max \left\{\mu_{1 i}, \mu_{1 j}\right\} \text { and } \gamma_{2 i j}=\min \left\{\gamma_{1 i}, \gamma_{1 j}\right\}
$$

$\forall i=1,2,3, \ldots, n$. But for the anti-complement $\widetilde{G_{A}}$,

$$
\widetilde{\mu_{21 j}}=1-\mu_{2 i j}+\max \left\{\mu_{1 i}, \mu_{1 j}\right\}=1-\mu_{2 i j}+\mu_{2 i j}=1,
$$

$\forall i, j=1,2,3, \ldots, n$. Now,

$$
\widetilde{\gamma_{21}}=1-\gamma_{2 i j}+\min \left\{\gamma_{1 i}, \gamma_{1 j}\right\}=1-\gamma_{2 i j}+\gamma_{2 i j}=1,
$$

$\forall i, j=1,2,3, \ldots, n$. Since the underlying graph of $G_{A}$ is complete,

$$
d_{G_{A}}\left(v_{i}\right)=\left(\sum_{k=1}^{n-1} 1, \sum_{k=1}^{n-1} 1\right)=(n-1, n-1)
$$

Thus $\widetilde{G_{A}}$ is $(n-1, n-1)$-regular.

Theorem 4.5. If $G_{A}=\langle V, E\rangle$ is an intuitionistic anti-fuzzy graph, then the underlying graph of $\widetilde{G_{A}}$ is complete.

Proof. Let $G_{A}=\langle V, E\rangle$ be an intuitionistic anti-fuzzy graph and $\widetilde{G_{A}}=(\bar{V}, \bar{E})$ be the anticomplement of $G_{A}$. So

(i) $\widetilde{V}=V$

(ii) $\widetilde{\mu_{11}}=\mu_{1 i}$ and $\widetilde{\gamma_{11}}=\gamma_{1 i}, \forall i=1,2,3, \ldots, n$

(iii) $\widetilde{\mu_{21}}=1-\mu_{2 i j}+\max \left\{\mu_{1 i}, \mu_{1 j}\right\}$ and $\widetilde{\gamma_{21 j}}=1-\gamma_{2 i j}+$ $\min \left\{\gamma_{1 i}, \gamma_{1 j}\right\}, \forall\left(v_{i}, v_{j}\right) \in E$.

When $\mu_{2 i j} \neq 0, \mu_{2 i j}>0, \forall\left(v_{i}, v_{j}\right) \in E \Rightarrow \widetilde{\mu_{211}}>0$

When $\mu_{2 i j}=0, \widetilde{\mu_{21}}=1+\max \left\{\mu_{1 i}, \mu_{1 j}\right\}>0$

Thus $\widetilde{\mu_{21}}>0, \quad \forall i=1,2,3, \ldots, n$.

When $\gamma_{2 i j} \neq 0, \gamma_{2 i j}>0, \forall\left(v_{i}, v_{j}\right) \in E \Rightarrow \widetilde{\gamma_{21}}>0$

When $\gamma_{2 i j}=0, \widetilde{\gamma_{21}}=1+\min \left\{\gamma_{1 i}, \gamma_{1 j}\right\}>0$

Thus $\widetilde{\gamma_{21}}>0, \quad \forall i=1,2,3, \ldots, n$

Therefore the underlying graph of $\overline{G_{A}}$ is complete.
Theorem 4.6. Let $G_{A}=\langle V, E\rangle$ be an intuitionistic anti-fuzzy graph. Then $\widetilde{G_{A}}=G_{A}$

Proof. Let $\widetilde{G_{A}}=(\widetilde{V}, \widetilde{E})$ be the anti-complement of intuitionistic anti-fuzzy graph $G_{A}=\langle V, E\rangle$. So

(i) $\widetilde{V}=V$

(ii) $\widetilde{\mu_{11}}=\mu_{1 i}$ and $\widetilde{\gamma_{11}}=\gamma_{1 i}, \forall i=1,2,3, \ldots, n$

(iii) $\widetilde{\mu_{21}}=1-\mu_{2 i j}+\max \left\{\mu_{1 i}, \mu_{1 j}\right\}$ and $\widetilde{\gamma_{21 j}}=1-\gamma_{2 i j}+$ $\min \left\{\gamma_{1 i}, \gamma_{1 j}\right\}, \forall\left(v_{i}, v_{j}\right) \in E$.

Considering the anti-complement $\widetilde{G_{A}}=\langle\widetilde{V}, \widetilde{E}\rangle$ of $\widetilde{G_{A}}=(\widetilde{V}, \widetilde{E})$. Thus

(i) $\widetilde{\widetilde{V}}=\widetilde{V}=V$

(ii) $\widetilde{\tilde{\mu}_{11}}=\widetilde{\mu_{11}}=\mu_{1 i}$ and $\widetilde{\gamma_{11}}=\widetilde{\gamma_{11}}=\gamma_{1 i}, \forall i=1,2,3, \ldots, n$

(iii)

$$
\begin{aligned}
\widetilde{\mu_{21}} & =1-\widetilde{\mu_{21}}, \widetilde{\max }\left\{\widetilde{\mu_{11}}, \widetilde{\mu_{1 j}}\right\} \\
& =1-\left[1-\mu_{2 i j}+\max \left\{\mu_{1 i}, \mu_{1 j}\right\}\right]+\max \left\{\widetilde{\mu_{11}}, \widetilde{\mu_{1 j}}\right\} \\
& =1-\left[1-\mu_{2 i j}+\max \left\{\mu_{1 i}, \mu_{1 j}\right\}\right]+\max \left\{\mu_{1 i}, \mu_{1 j}\right\} \\
& =\mu_{2 i j}, \quad \forall\left(v_{i}, v_{j}\right) \in E \\
\widetilde{\gamma_{21}} & \left.=1-\widetilde{\gamma_{21}}+\min \left\{\widetilde{\gamma_{11}}, \widetilde{\gamma_{1}}\right]\right\} \\
& =1-\left[1-\gamma_{2 i j}+\min \left\{\gamma_{1 i}, \gamma_{1 j}\right\}\right]+\min \left\{\widetilde{\gamma_{11}}, \widetilde{\gamma_{1 j}}\right\} \\
& =1-\left[1-\gamma_{2 i j}+\min \left\{\gamma_{1 i}, \gamma_{1 j}\right\}\right]+\min \left\{\gamma_{1 i}, \gamma_{1 j}\right\} \\
& =\gamma_{2 i j}, \quad \forall\left(v_{i}, v_{j}\right) \in E
\end{aligned}
$$

Definition 4.7. An intuitionistic anti-fuzzy graph $G_{A}$ is selfanti-complementary if $G_{A}=\widetilde{G_{A}}$.

Example 4.8. See Fig. 13 and 14 .

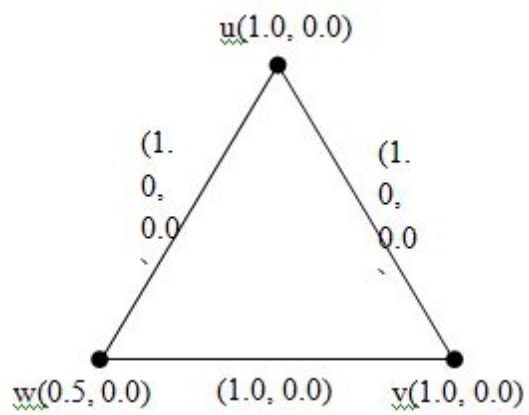

Figure 13. Intuitionistic anti-fuzzy graph $G_{A}$

Theorem 4.9. Let $G_{A}=\langle V, E\rangle$ be an intuitionistic anti-fuzzy graph. If $G_{A}$ is self-anti-complementary then,

$$
\mu_{2 i j}=\frac{1}{2}\left[1+\max \left\{\mu_{1 i}, \mu_{1 j}\right\}\right]
$$

and

$$
\gamma_{2 i j}=\frac{1}{2}\left[1+\min \left\{\gamma_{1 i}, \gamma_{1 j}\right\}\right] .
$$




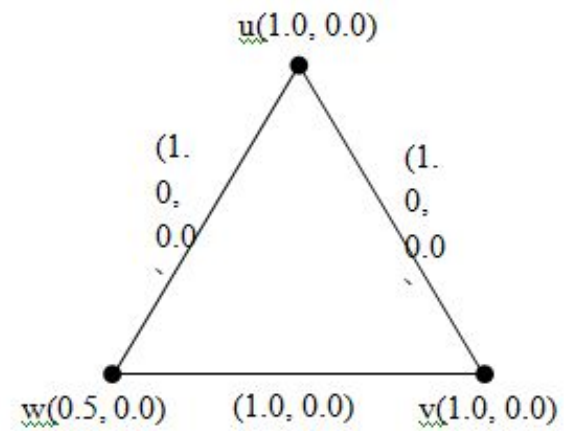

Figure 14. Anti-complement graph $\widetilde{G_{A}}$

Proof. Let $G_{A}=\langle V, E\rangle$ be a self-anti-complementaryintuitionistic anti-fuzzy graph. Hence $G_{A}=\widetilde{G_{A}}$. Therefore, $\left(\mu_{1}, \gamma_{1}\right)=$ $\left(\widetilde{\mu_{1}}, \widetilde{\gamma_{1}}\right)$ and $\left(\mu_{2}, \gamma_{2}\right)=\left(\widetilde{\mu_{2}}, \widetilde{\gamma_{2}}\right)$. Thus

$$
\begin{aligned}
\widetilde{\mu_{21}} & =1-\mu_{2 i j}+\max \left\{\mu_{1 i}, \mu_{1 j}\right\} \\
\mu_{2 i j} & =1-\mu_{2 i j}+\max \left\{\mu_{1 i}, \mu_{1 j}\right\} \\
2 \mu_{2 i j} & =1+\max \left\{\mu_{1 i}, \mu_{1 j}\right\} \\
\mu_{2 i j} & =\frac{1}{2}\left[1+\max \left\{\mu_{1 i}, \mu_{1 j}\right\}\right]
\end{aligned}
$$

again

$$
\begin{aligned}
\widetilde{\gamma_{21}} & =1-\gamma_{2 i j}+\min \left\{\gamma_{1 i}, \gamma_{1 j}\right\} \\
\gamma_{2 i j} & =1-\gamma_{2 i j}+\min \left\{\gamma_{1 i}, \gamma_{1 j}\right\} \\
2 \gamma_{2 i j} & =1+\min \left\{\gamma_{1 i}, \gamma_{1 j}\right\} \\
\gamma_{2 i j} & =\frac{1}{2}\left[1+\min \left\{\gamma_{1 i}, \gamma_{1 j}\right\}\right]
\end{aligned}
$$

\section{Application of intuitionistic anti-fuzzy graph}

Intuitionistic anti-fuzzy graph is applicable in many altered situations of human life. This intuitionistic anti-fuzziness can be functional in 2020 world disaster coronavirus disease or Covid-19. For this, consider $\mathrm{n}$ persons and represent them by $n$ different nodes of an intuitionistic anti-fuzzy graph such that the membership and non-membership values are lying between 0 and 1 , both inclusive. The membership value can be defined according to the person who is having very mild symptoms or pre-symptomatic coronavirus disease, Covid-19, to ferocious symptoms. The immunity power of a person is graded as the non-membership value of corresponding vertex. Construct edges on the basis of physical interactions or communications or close contact between two persons such that the membership value is bigger or equal to the maximum of the membership values of them and in the similar way non-membership value can vary due to close contact and membership value. The physical communication between persons increases the intensity of the affection of corona virus, which leads to increase the membership value of the vertex and due to this the immunity power of the person decreases, which leads to the non-membership value of the vertex. These changes in the affection of corona virus and immunity power can be represented by arcs between vertices of an intuitionistic anti-fuzzy graph.

Based on these information it is possible to construct an intuitionistic anti-fuzzy graph and illustrate the situation thoroughly.

Consider the following intuitionistic anti-fuzzy graph (figure 15) for 5 persons who are communicating each other to transfer the coronavirus. In this intuitionistic anti-fuzzy graph

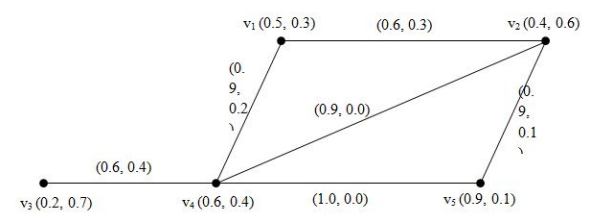

Figure 15. Intuitionistic anti-fuzzy graphG ${ }_{A}$

$G_{A}, v_{1}$ is a vertex which represent a person $P_{1}$ where 0.5 is the membership value for the rate of affectedness of coronavirus disease according to the common symptoms and 0.3 is the non-membership value for the quantitative representation of immunity power of his body in the current situation. Thus in $v_{1}$ for the person $P_{1}, \mu_{1}=0.5$ and $\gamma_{1}=0.3$. If the value of $\mu_{1}$ is increased, then automatically the value of $\gamma_{1}$ will decrease by the definition of an intuitionistic anti-fuzzy graph according to the criteria $0 \leq \mu_{1}+\gamma_{1} \leq 1$ for a vertex. The rate of spread of this virus on communication or mutual contacts can be represented by an edge between two vertices. The membership values $\mu_{1}$ or $\mu_{2}$ are decided on the basis of the intensity of common symptoms like persistent cough, continuous fever of more than 102 degrees, difficulty in breathing, persistent pain or pressure in the chest, mental confusion or inability to arouse the patient, somnolence and poorfeeding in children, seizures, decreased urine output, persistent or worsening of initial symptoms beyond 72 hours and developing bluish discoloration of lips or face.

Joining two vertices in this intuitionistic anti-fuzzy graph would cause to the increase of infection and increase in death rate due to the disease. It is possible to isolate the vertices or human beings using different mode of quarantine techniques for a large number of humans by taking the domination of intuitionistic anti-fuzzy graphs.Here it is easy to break the multiple connected domination followed by connected strong domination using the theory of split and strong split dominations in intuitionistic anti-fuzzy graphs [12]. For that it is necessary to identify the strong arcs and the strong domination set. Isolation of vertices in an isolated intuitionistic anti-fuzzy graph prevent the increase in membership value of edges or prevent to transfer virus from vertex to vertex and hence which results the decrease the rate of spread of the virus from the society.

Consider an example to represent this situation by using intuitionistic anti-fuzzy graphs:

Here $\mathrm{c}$ is a vertex in place of a person having quantitative 


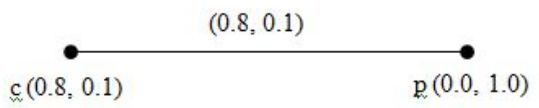

Figure 16. Intuitionistic anti-fuzzy graph $G_{A}$

representation of corona virus affection by common symptoms as 0.8 and his contemporaneous immunity power as 0.1 . Again $\mathrm{p}$ is a vertex in place of a second person having quantitative representation of corona virus affection by common symptoms as 0.0 and his contemporaneous immunity power as 1.0. In this case second person $\mathrm{p}$ is having no infection of corona virus at this stage and his health condition is perfectly good, so the immunity power is at the peak. If these peoples are in close contact to each other, then the person $\mathrm{p}$ may infected by corona virus and the rate of infection may bigger or equal to 0.8 , so as the immunity power may reduce accordingly.

So intuitionistic anti-fuzzy graph has significant application in health and medical field especially in communicable diseases like covid-19 pandemic. It is possible to study and analyze the infection rate of such viruses in different areas of our society by using intuitionistic anti-fuzzy graphs and which will be helpful to adopt proper remedial measures to remove the disease from the surroundings.

\section{Conclusion}

The concept of intuitionistic anti-fuzzy graphs and their special cases like intuitionistic anti-fuzzy sub graphs, vertex $(\mu, \gamma)$-degrees and cardinality and strong intuitionistic antifuzzy graphs were introduced. Complement of strong intuitionistic anti-fuzzy graph has been defined and develop some results on it. Anti-complement is applied on intuitionistic antifuzzy graphs and some theorems and results are found with proof. A significant application of intuitionistic anti-fuzzy graph on medical ground is explained in the infectious circumstances of pandemic covid-19 is described. The theory of intuitionistic anti-fuzzy graph has more applications in efficiency management, communication networks, information technology, pattern clustering, image retrieval and so on. In future, it is anticipated to do these perceptions on the other extension of intuitionistic anti-fuzzy graphs.

\section{References}

[1] K. T. Atanassov, Intuitionistic Fuzzy Sets: Theory and Applications, Physica, New York, 1999.

[2] P. Chountas, M. Alzebdi, A. Shannon, K. Atanassov, On intuitionistic fuzzy trees, Notes on Intuitionistic Fuzzy Sets, 15(2)(2009), 30-32.

[3] A. Kaufmann, Introduction to the Theory of Fuzzy Subsets, Vol.1, Academic press, New York, 1975.

[4] M. G. Karunambigai, S. Sivasankar, K. Palanivel, Secure Edge Domination and Vertex Edge Domination in Intuitionistic Fuzzy Graphs, International Journal of Mathematical Archive, 9(1)(2018), 190-196.
[5] J. N. Mordeson, P. S. Nair, Fuzzy Graphs and Fuzzy Hypergraphs, Physica-Verlag, Heidelberg, New York (2000).

[6] Muhammad Akram, Anti Fuzzy Structures on graphs, Middle East Journal of Scientific Research, 11(12)(2012), 1641-1648.

[7] R. Muthuraj, A. Sasireka, On anti fuzzy graph, Advances in Fuzzy Mathematics, 12(5)(2017), 1123-1135.

[8] R. Muthuraj, A. Sasireka, Some Characterization on Operations of Anti Fuzzy Graphs, International Conference on Mathematical Impacts in Science and Technology, (MIST-17), November 2017, IJRASET, 109 - 117.

[9] R. Muthuraj, S. Sujith and V. V. Vijesh, Operations on intuitionistic anti fuzzy graphs, International Journal of Recent Technology and Engineering, (IJRTE), ISSN: 2277-3878, 8(2019), 1098 - 1103.

[10] R. Muthuraj, V. V. Vijesh and S. Sujith, Domination on intuitionistic anti fuzzy graphs, Advances in Mathematics: Scientific Journal, 8, ISSN 1857-8365 printed version and ISSN 1857-8438 electronic version (2019)

[11] R. Muthuraj, V. V. Vijesh and S. Sujith, Split and Strong Split Dominations in Intuitionistic Anti-Fuzzy Graphs, Solid state technology, 63(6)(2020), 20989-20999.

[12] A. Nagoor Gani, S. S. Begum, Degree, order and size in intuitionistic fuzzy graphs, Int. J. Algorithms, Computing and Mathematics, 3(3)(2010), 11-16.

[13] R. Parvathi, M. G. Karunambigai, intuitionistic fuzzy graphs, Computational Intelligence, Theory and Applications: International Conference 9th Fuzzy Days in Dortmund, Germany, Sept. 18-20, 2006 Proceedings, (2006) 139-150.

[14] R. Parvathi, M. G. Karunambigai, K. Atanassov, operations on intuitionistic fuzzy graphs, Proceedings of IEEE International Conference on Fuzzy Systems(FUZZ-IEEE), (2009), 1396-1401.

[15] A. Rosenfeld, Fuzzy graphs, in Fuzzy Sets and Their Applications, Academic Press, New York, NY, USA, 1975.

[16] R. Seethalakshmi, R. B. Gnanajothi, Operations on antifuzzy graph, Mathematical Sciences International Research Journal, 5(2)(2016), 210-214.

[17] A. Shannon, K. Atanassov, On a generalization of intuitionistic fuzzy graphs, Notes on intuitionistic fuzzy sets, 12(1)(2006), 24-29.

[18] M. S. Sunitha, A. Vijayakumar, Complement of a fuzzy graph, Indian J. Pure Appl. Math, 33(9)(2002), 14511464.

[19] V. V. Vijesh, R. Muthuraj, Some Characteristics on Join of Intuitionistic Fuzzy Graphs, IOSR Journal of Mathematics (IOSR-JM), e-ISSN:2278-5728, p-ISSN: 2319-765X, $23-31$.

[20] L. A. Zadeh, Fuzzy Sets, Information Sciences, 8(1965), 338-353. 
Malaya Journal of Matematik

ISSN(O):2321 - 5666

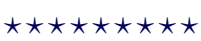

